https://helda.helsinki.fi

\title{
Laccase/TEMPO oxidation in the production of mechanically strong arabinoxylan and glucomannan aerogels
}

\section{Parikka, Kirsti Maria}

2017

Parikka , K M , Nikkilä , I E , Pitkänen , L M , Ghafar , A , Sontag-Strohm , T S \& Tenkanen , T M 2017 , ' Laccase/TEMPO oxidation in the production of mechanically strong arabinoxylan and glucomannan aerogels ' , Carbohydrate Polymers , vol. 175 , pp. 377-386 . https://doi.org/10.1016

http://hdl.handle.net/10138/308665

https://doi.org/10.1016/j.carbpol.2017.07.074

cc_by_nc_nd

acceptedVersion

Downloaded from Helda, University of Helsinki institutional repository.

This is an electronic reprint of the original article.

This reprint may differ from the original in pagination and typographic detail.

Please cite the original version. 


\title{
Laccase/TEMPO oxidation in the production of mechanically strong arabinoxylan and glucomannan aerogels
}

Kirsti Parikka*, Ida Nikkilä, Leena Pitkänen, Abdul Ghafar, Tuula Sontag-Strohm, Maija Tenkanen Department of Food and Environmental Sciences, P.O. Box 66 (Agnes Sjöbergin katu 2), FI-00014 University of Helsinki, Finland

*Email: kirsti.parikka@helsinki.fi

\begin{abstract}
New wheat arabinoxylan and konjac glucomannan hydrogels and aerogels were prepared by hemiacetal crosslinking induced by laccase/TEMPO (2,2,6,6-tetramethylpiperidine-1oxyl) -catalyzed oxidation, which converts selectively primary hydroxyl groups to aldehydes. The degree of oxidation of the product aldehydes was ca. $10 \%$ of the total carbohydrates of the polysaccharides, and the determination of storage and viscous moduli of the oxidized samples showed that they had formed true hydrogels. Two freezing methods for the hydrogels, conventional freezing and ice crystal templating, were investigated in aerogel production, and especially the ice crystal templated products were mechanically very strong in compression test against the ice crystal growth direction. The compressive moduli were ca. $1200 \mathrm{kPa}$ for wheat arabinoxylan aerogels and ca. $650 \mathrm{kPa}$ for konjac glucomannan aerogels. Morphological study with scanning electron microscope revealed the inner structure of aerogels. Ice crystal templated konjac glucomannan aerogel formed round pores with a diameter of ca. 50-100 $\mu \mathrm{m}$. Arabinoxylan aerogel consisted of long and narrow pores with length of a few hundred $\mu \mathrm{m}$ and width of 50-100 $\mu \mathrm{m}$, which had formed in the direction of ice crystal formation. Konjac glucomannan and wheat arabinoxylan are approved food grade materials, and particularly wheat arabinoxylan is highly interesting, as it can be obtained from cereal processing side streams - thus the novel products have potential in various applications including e.g. food, food packaging, and pharmacological fields.
\end{abstract}

Keywords wheat arabinoxylan, konjac glucomannan, guar galactomannan, TEMPO, laccase, oxidation, aldehyde, hydrogel, aerogel 


\section{Introduction}

Polysaccharides are renewable, non-toxic, and biodegradable materials and thus highly valuable resources for modern industry, which demands advanced materials having potential to replace synthetic plastics. Polysaccharides can be feasibly obtained from side-streams of agriculture and forestry industry, but they have not yet been utilized to their full possibilities. The functionality of native polysaccharides can be improved by enzymatic and chemical modifications, affecting the structure of the polysaccharides, for example, by mild degradation, by addition of new functional groups, or by creating crosslinks.

Aerogels are lightweight and mechanically strong porous materials, which have large surface area. They can be formed in various shapes and sizes by the removal of liquid from a gel, e.g. evaporation of water from a hydrogel. Conventionally, aerogels have been prepared from inorganic materials, such as silica, or organic materials, for example, resorcinol-formaldehyde polymers (phenolic resins), which have been utilized e.g. as thermal insulators [Mulik and Sotiriou-Leventis, 2011]. The concept of polysaccharides as a resource for aerogel production is relatively new. For example, cellulose aerogels (aerocellulose) are ultra-light and highly porous materials, which have densities comparable to silica aerogels [Buchtová and Budtova, 2016; Sescousse et al., 2011]. New results have been obtained on pectin-based or alginate-lignin aerogels, having potential in drug release applications [Tkalec et al., 2015; Quraishi et al., 2015] or as thermal insulators [Rudaz et al., 2014], and cellulose aerogel has been studied in tissue engineering [Pircher et al., 2015]. Aerogels prepared of cellulose, starch, different hemicelluloses, and marine polysaccharides have been reviewed in the aspect of advanced food applications [Mikkonen et al., 2013]. Biomedical applications of aerogels, including polysaccharide aerogels, have also been reviewed [Stergar \& Maver, 2016].

Previously, we have reported the targeted enzymatic oxidation of various polysaccharides by galactose oxidase (EC 1.1.3.9), producing polysaccharides with galactoaldehyde functionality [Parikka et al., 2010]. The formed aldehydes are capable to crosslink the polysaccharide chains via hemiacetal bonds, thus forming elastic hydrogels and mechanically strong water-insoluble aerogels [Parikka et al., 2012; Mikkonen et al., 2014]. Due to the high selectivity of galactose oxidase [Parikka et al., 2015], this technique is limited to galactose-containing polysaccharides, such as galactomannans and galactoxyloglucan, where the galactose units are attached as side groups with free C-6.

To modify a wider variety of polysaccharides, such as, wheat arabinoxylan (AX) and konjac glucomannan (KGM), we focused on the possibility to create the crosslinking aldehydes by another, chemo-enzymatic method. The combination of laccase, copper-containing oxidoreductase (EC 
1.10.3.2) and TEMPO (2,2,6,6-tetramethylpiperidine-1-oxyl) produced aldehyde derivatives of polysaccharides in slightly acidic reaction conditions [Jausovec, 2015]. In TEMPO-mediated oxidation in general, primary alcohols are converted to aldehydes and further to carboxylic acids. TEMPO acts as primary oxidant and requires a secondary oxidant, which regenerates TEMPO in the catalytic cycle. Reaction conditions determine whether the products are aldehydes or carboxylic acids [Tojo and Fernandez, 2007]. In the case of carbohydrates, the TEMPO-mediated reaction, often $\mathrm{NaBr} / \mathrm{NaClO}$ as the secondary oxidant, has been utilized in the preparation of uronic acid derivatives both from cold water soluble and cold water insoluble polysaccharides such as starch, inulin, amylodextrin, pullulan, potato amylose, potato amylopectin, chitin, chitosan, and dextran [Bragd 2004]. Cellulose has also been oxidized by similar methods [Isogai et al., 2011]. TEMPOmediated modifications of polysaccharides have been recently reviewed [Pierre et al., 2017].

The use of laccase as the secondary oxidant in the regeneration of TEMPO has previously been studied in the oxidation of cellulose [Viikari, 1999; Jausovec, 2015], pullulan [Jetten 2000], and certain galactomannans [Lavazza et al., 2011; Merlini et al., 2015; Rossi et al., 2016]. Laccase itself has a wide range of substrates, such as, substituted mono- and polyphenols, aromatic amines and thiol compounds [Madhavi and Lele, 2009]. Especially, laccase from Trametes versicolor is suitable for the secondary oxidant in modification of mannan polysaccharides, as it does not have mannanase side activity, which other commercially available laccases from Thielavia arenaria and Cerrena unicolor have [Lavazza et al., 2011]. The ability of laccase to oxidize TEMPO is dependent on the redox potential of the enzyme and thus e.g. laccase from Myceliophthora thermophilia was not suitable [Lavazza et al., 2011; Rodgers et al., 2010]. Laccase (e.g. from Trametes hirsuta) has also been studied as an additive in wheat doughs, where it is expected to catalyze the oxidation and thus dimerization of esterified ferulic acid units in AX, crosslinking and gelling the polymers. However, it might also induce radical-catalyzed degradation of the crosslinked AX [Selinheimo et al., 2006].

KGM hydrogel is traditionally utilized as dietary food in Japan. It is formed by deacetylation of native KGM in alkaline conditions $(\mathrm{pH}>11)$, and a concentration of $0.5 \%(\mathrm{w} / \mathrm{v})$ is high enough for formation of a chewy gel [Takigami, 2009]. AX can be isolated from cereal processing side streams. The viscosity of native AX depends on concentration and $M_{\mathrm{w}}$, and concentrated solutions exhibit shear thinning at high shear rates, behaving thus as pseudoplastic materials or weakly elastic gels crosslinked by hydrogen bonds [Izydorczyk, 2009]. Some reports exist on aerogels utilizing KGM and AX. Aerogels containing KGM have been prepared e.g. by combining KGM with clay [Li et al., 2014] or glyceryl monostearate [Ni et al., 2016]. AX aerogels have been prepared 
utilizing laccase or horse radish peroxidase induced dimerization of the feruloyl units, present in the polysaccharide, as the crosslinking technique [Escalante et al., 2013; Iravani et al.; 2011].

This is the first report on the oxidation of AX and KGM by the laccase/TEMPO system, producing the aldehyde derivatives, and hemiacetal-crosslinked hydrogels and aerogels of these polysaccharides. Aerogels were prepared by lyophilization of hydrogels after either conventional freezing or ice crystal templating. The degree and site of oxidation were determined by GC-MS. The rheological properties of hydrogels and molar mass of the oxidized polysaccharides were studied. The density, water absorption capacity, mechanical properties, and morphology of aerogels were investigated, and the residual TEMPO in the products was determined. Guar galactomannan (GM) aerogels were prepared with the same method for comparison of the properties of the products to the properties of aerogels prepared by of galactose oxidase -catalyzed oxidization [Mikkonen et al., 2014].

\section{Materials and methods}

\subsection{Polysaccharides, laccase and TEMPO}

Guar galactomannan (GM, G4129), laccase (Trametes versicolor, 38429, $0.5 \mathrm{U} / \mathrm{mg}$ ) and TEMPO were purchased from Aldrich (St. Louis, MO, USA). Konjac glucomannan (KGM, Luxara-208-1) was obtained from Arthur Branwell \& Co. Ltd. (Epping, Essex, GB). Wheat arabinoxylan (AX, 120707, high viscosity), was purchased from Megazyme (Wicklow, Ireland).

\subsection{Oxidation of polysaccharides and preparation of aerogels}

Polysaccharides were dissolved in $12.5 \mathrm{mM}$ sodium citrate buffer (pH 5.0) at RT. The concentrations were $1 \% \mathrm{w} / \mathrm{v}$ for GM and $\mathrm{KGM}$ and $2 \% \mathrm{w} / \mathrm{v}$ for $\mathrm{AX}(1 \% \mathrm{w} / \mathrm{v}$ solution of $\mathrm{AX}$ did not form hydrogel after oxidation). After stirring for $1 \mathrm{~h}$, laccase and TEMPO were added with the dosages of $200 \mathrm{U} / \mathrm{g}$ of polysaccharide and $50 \mathrm{mg} / \mathrm{g}$ of polysaccharide, respectively. Stirring was continued in an open vessel. After $24 \mathrm{~h}$, hydrogel had formed, and the enzyme was inactivated by placing the reaction vessel into boiling water bath for $15 \mathrm{~min}$.

The hydrogels were allowed to cool and then transferred into cubical Petri dishes utilizing spatula. To prepare samples frozen by ice crystal templating, the Petri dishes were placed onto a $\mathrm{CO}_{2}$ ice plate in an EtOH bath. After 0.5-1 h the hydrogel was frozen, the samples removed from the bath and kept at $-70^{\circ} \mathrm{C}$ for at least one day before lyophilization. To prepare samples frozen by conventional freezing, the Petri dishes containing the hydrogels were placed at $-20{ }^{\circ} \mathrm{C}$ and after 
being frozen, kept at $-70^{\circ} \mathrm{C}$ as the ice crystal templated samples. Water in the frozen gels was removed by lyophilization.

\subsection{Determination of residual TEMPO in the products}

Aerogel cubes (ca. 10 specimens) were crushed, combined and stirred in $\mathrm{CH}_{2} \mathrm{Cl}_{2}(65 \mathrm{ml})$ for $72 \mathrm{~h}$. The mixture was filtered, washed and the solvent concentrated up to $1 \mathrm{ml}$. The sample was directly analyzed by GC-MS and the amount of TEMPO calculated based on a standard curve.

\subsection{Analysis of the degree and site of oxidation}

Samples (ca. $0.5 \mathrm{ml} ; 5$ or $10 \mathrm{mg}$ of polysaccharide) were taken from the hydrogels after laccase had been inactivated. A previously developed GC-MS method was used to determine the degree of oxidation [Parikka et al., 2010]. The aldehydes formed in the oxidation were reduced back to alcohols with $\mathrm{NaBD}_{4}$ introducing deuterium labels. The samples were methanolyzed, silylated and analyzed by GC-MS. For hexoses (galactose, glucose, mannose), the degree of oxidation (DO) was then calculated from the difference between m/z 361 and m/z 362 ions in their mass spectra, $\mathrm{m} / \mathrm{z}$ 361 representing unreacted starting material and m/z 362 representing the oxidized (and deuterated) product. Correspondingly, for the pentose arabinose, the structurally similar ion pair $\mathrm{m} / \mathrm{z} 259$ and $\mathrm{m} / \mathrm{z} 260$ represented the unoxidized and oxidized forms. Thus, in addition to the determination of DO, the GC-MS technique revealed the site of oxidation, i.e. which monosaccharide units of the polysaccharide were oxidized.

\subsection{Size Exclusion Chromatography}

Samples (ca. $0.5 \mathrm{ml} ; 5$ or $10 \mathrm{mg}$ of polysaccharide) were taken from the hydrogels after the inactivation of the enzyme, reduced with $\mathrm{NaBH}_{4}$ as described earlier [Parikka et al., 2010], and diluted in $0.1 \mathrm{M} \mathrm{NaNO}_{3}$ to ca. $1 \mathrm{mg} / \mathrm{ml}$ concentration. High performance size exclusion chromatography (HPSEC) analysis of the samples was carried out using $0.1 \mathrm{M} \mathrm{NaNO}_{3}$ as an eluent. Prior to HPSEC analysis, samples $(\mathrm{c}=1 \mathrm{mg} / \mathrm{ml})$ were filtered with $0.45 \mu \mathrm{m}$ syringe filters (GHP Acrodisc 13, Pall Corp., Ann Arbor, MI, U.S.A.). The HPSEC equipment consisted of an integrated autosampler and pump module (GPCmax, Viscotek Corp., Houston, TX, U.S.A.), a combined light scattering and viscometric detector (270 Dual Detector, Viscotek Corp.), and a refractive index (RI) detector (VE 3580, Viscotek Corp.). Two linear type Shodex columns were used (OHpak SB-806M HQ, $8 \times 300 \mathrm{~mm}$, exclusion limit $2 \times 107$, Showa Denko, Ogimachi, Japan) with a guard column (OHpak SB-6, $4.6 \times 10 \mathrm{~mm})$. Light scattering detector $(\lambda 0=670 \mathrm{~nm})$ included two scattering angles: $7^{\circ}$ (low angle light scattering, LALS) and $90^{\circ}$ (right angle light scattering, RALS). The flow 
rate was $1 \mathrm{ml} / \mathrm{min}$ and injection volume $100 \mu \mathrm{l}$. The molar masses were calculated based on the light scattering/viscometry method using the approximate $\mathrm{dn} / \mathrm{dc}$ value of $0.15 \mathrm{~mL} / \mathrm{g}$ for all the samples. The combined light scattering and viscometric detector and RI detector were calibrated with a pullulan narrow standard ( $\mathrm{Mw}=212000 \mathrm{~g} / \mathrm{mol}$, Polymer Laboratories, Shropshire, U.K.). Intrinsic viscosities $([\eta])$ were calculated based on the viscosity signals obtained from the viscometric detector. The calculations regarding weight average molar mass $(\mathrm{Mw})$, intrinsic viscosity and hydrodynamic radius (Rh) were done using OmniSEC 4.5 software (Viscotek Corp.).

\subsection{Viscoelastic characterization of hydrogels}

The rheological analysis of hydrogels was performed in triplicates using a controlled stress rheometer (Rheostress RS600, Thermo Haake, Germany). The measurement was performed directly after hydrogel formation ( $24 \mathrm{~h}$ reaction). The reference samples of native polysaccharides prepared and handled similar way but without laccase and TEMPO. The measuring geometry was parallel plate-plate with diameter of $35 \mathrm{~mm}$ and the gap between the two plates $1.0 \mathrm{~mm}$. Viscoelastic moduli were determined as a function of frequency sweep and in frequency range 0.03-10 Hz. The measurements were performed in the linear viscoelastic range determined by a stress sweep. Temperature was regulated with a Haake water-cooled immersion circulator (DC50-K10) and kept at $20^{\circ} \mathrm{C}$.

\subsection{Density of aerogels}

The density of cubic aerogel samples was determined by utilizing glass beads $(0.2 \mathrm{~mm}$ diameter, $1.68 \mathrm{~g} \mathrm{~cm}^{-3}$ ). The cube (weight $=\mathrm{w}_{\mathrm{s}}$ ) was placed into a glass container of known volume and weight $\left(\mathrm{w}_{\mathrm{d}}\right)$, and the container filled with the beads. The weight was recorded (container, sample, and beads $\left.=\mathrm{w}_{\mathrm{dsb}}\right)$ and the weight of the beads $\left(\mathrm{w}_{\mathrm{b}}\right)$ calculated by subtracting: $\mathrm{w}_{\mathrm{b}}=\mathrm{w}_{\mathrm{dsb}}-\mathrm{w}_{\mathrm{d}}-\mathrm{w}_{\mathrm{s}}$. The volume of the beads $\left(\mathrm{v}_{\mathrm{b}}\right)$ was then obtained by $\mathrm{v}_{\mathrm{b}}=\mathrm{w}_{\mathrm{b}} / 1.68 \mathrm{~g} \mathrm{ml}^{-} 1$. The density of the aerogel sample $\left(\rho_{\mathrm{s}}\right)$ was calculated by dividing $\mathrm{w}_{\mathrm{s}}$ by the reminder of $\mathrm{v}_{\mathrm{b}}$ subtracted from $\mathrm{v}_{\mathrm{d}}\left(\rho_{\mathrm{s}}=\mathrm{w}_{\mathrm{s}} /\left(\mathrm{v}_{\mathrm{d}}-\right.\right.$ $\left.\mathrm{v}_{\mathrm{b}}\right)$ ). The determination was conducted for three cubic samples and the average represented the density.

\subsection{Texture analysis of aerogels}

Two consecutive cycles of compression were performed at RT for the cubic aerogel samples (kept at $50 \%$ relative humidity for 3 days) using Texture Analyser TA-XT2i (Stable Microsystems, Godalming, UK). Ten cubic sample replicates of approximate width and thickness of $17 \mathrm{~mm}$ but slightly varying height were tested in vertical direction using $36 \mathrm{~mm}$ DIA aluminum cylinder with 
contact area of $1017.88 \mathrm{~mm}^{2}$, force of $5.0 \mathrm{~kg}$ or $30.0 \mathrm{~kg}$ with trigger forces of ca. $0.010 \mathrm{~N}$ and 0.050 $\mathrm{N}$, respectively, and $35 \%$ compression of each sample. Pre-test speed was $1.0 \mathrm{~mm} / \mathrm{s}$, test speed 2.0 $\mathrm{mm} / \mathrm{s}$, and post-test speed $2.0 \mathrm{~mm} / \mathrm{s}$. From the obtained force-time profile and stress-strain curve, hardness, resilience and compressive moduli were determined.

\subsection{Water absorption of aerogels}

The cubic aerogel samples were weighed and placed into decanter glasses containing $50 \mathrm{ml}$ of water. The water was magnetically stirred at RT. The amount of absorbed water in the cubes was weighed after 1, 3, and $24 \mathrm{~h}$. The cubes were taken from the decanter glasses and rolled quickly on a wipe to remove excess water from the surface. The weight of the dry cubes was subtracted from the weight of the wet cubes to obtain the amount of absorbed water. The average of triplicate samples was calculated.

\subsection{Morphology of aerogels}

Blocks of ca. 3-5 mm were cut from the cubic aerogel samples by a scalpel to study the crosssections and side views from both ice crystal templated and conventionally frozen samples. The sample blocks were coated with Pt (Quorun 150, Quorum Technologies, Laughton, UK). They were imaged on FEI Quanto 250 FEG scanning electron microscope (FEI, Hillsboro, OR, USA).

\section{Results}

\subsection{Oxidation of polysaccharides}

AX and KGM were selected for this study: KGM is already utilized in food applications, and AX has high potential due to the possibility to extract it from cereal processing side streams. Also, they do not contain galactosyl units and thus cannot be oxidized by galactose oxidase. They represent two different structural types: in AX the sugar units carrying the primary hydroxyl groups are located as side groups and in KGM primary hydroxyls are attached to backbone sugar units (Figure $1 \mathrm{~A})$. AX is xylan $(\beta-(1 \rightarrow 4)-\mathrm{D}-\mathrm{Xyl} p)$ carrying arabinofuranosyl units (ca. $30 \%)$ attached either to C-2 and/or C-3 of xylosyl residues [Virkki et al., 2008]. KGM is glucomannan having no single residue branches $(\beta-(1 \rightarrow 4)-D-M a n p / G l c p$; Man 50-60\%, Glc 40-50\%) [Takigami, 2009]. The oxidation of GM was also studied. Earlier, we have investigated the hydrogel and aerogel formation of GM by hemiacetal crosslinking with galactose oxidase -catalyzed oxidation [Parikka et al., 2010; Mikkonen et al., 2014]. GM can be also oxidized by TEMPO/laccase system [Lavazza et al., 2011; Merlini et al., 2015]. GM is mannan $(\beta-(1 \rightarrow 4)-\mathrm{D}-\mathrm{Man} p)$, having galactopyranosyl side groups (33- 
40\%) attached to the position C-6 of the mannosyl units [Vielinga, 2009]. The molar masses of AX, KGM, and GM are ca. 320-340 kDa [Pitkänen et al., 2009], ca. 1000-2000 kDa [Nieto, 2014], and ca. 1800-2200 kDa [Vielinga, 2009; Pitkänen et al., 2011], respectively.

Preliminary experiments showed that AX-ox did not form elastic hydrogel at $1 \%(\mathrm{w} / \mathrm{v})$ concentration, and thus $2 \%(\mathrm{w} / \mathrm{v})$ solution of $\mathrm{AX}$ was prepared for the oxidation. KGM and GM were oxidized at $1 \%(\mathrm{w} / \mathrm{v})$ concentration as $2 \%$ was not manageable in these cases. The highest obtained degree of oxidation (DO; Table 1) of total carbohydrates was approximately similar for KGM-ox and AX-ox, 11-12\%. Surprisingly more glucosyl (ca. $18 \%$ ) than mannosyl residues (ca. $5 \%$ ) were oxidized in KGM-ox. In AX-ox, DO of the arabinosyl side residues was ca. $20 \%$. Relatively high, ca. 15\%, oxidation of glucosyl residues was observed in the AX-ox product, due to the presence of a glucan impurity (5-8 \% glucose content) [Virkki et al., 2008], which might have a role in the crosslinking of the polysaccharide chains as well. An example of the oxidation reaction is shown in Figure $1 \mathrm{~B}$.

GM-ox was oxidized with approximately similar DO of total carbohydrates as KGM-ox and AX-ox. Most of the oxidation had occurred in the galactosyl units (DO $25 \%$ ), which exist as side groups attached to the mannan backbone, and as in the case of KGM, mannosyl residues were poorly oxidized (ca. $3 \%$ ).

Table 1. The degree of oxidation $(\%)$ of total carbohydrates in the polysaccharide (Total), and the oxidized monosaccharide residues of the polysaccharides wheat arabinoxylan (AX-ox), konjac glucomannan (KGM-ox), and guar galactomannan (GM-ox).

\begin{tabular}{lccccc}
\hline & Total & Arabinose & Glucose & Mannose & Galactose \\
\hline AX-ox & 11.7 & 20.1 & 15.2 & & \\
\hline KGM-ox & 11.3 & & 18.1 & 4.8 & \\
\hline GM-ox & 10.5 & & & 2.7 & 25.0 \\
\hline
\end{tabular}


A

AX

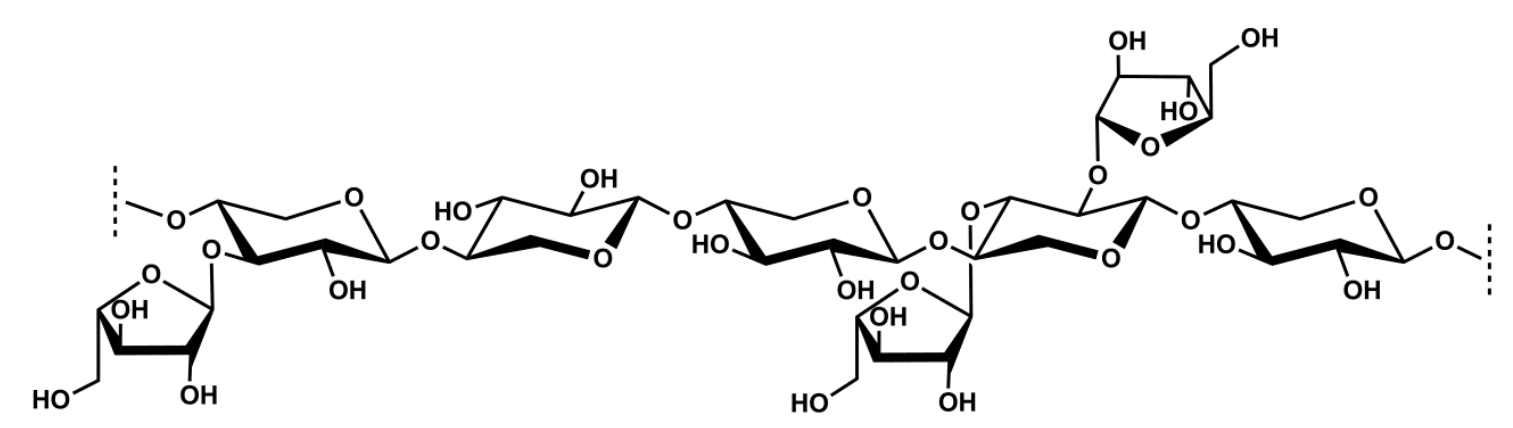

KGM

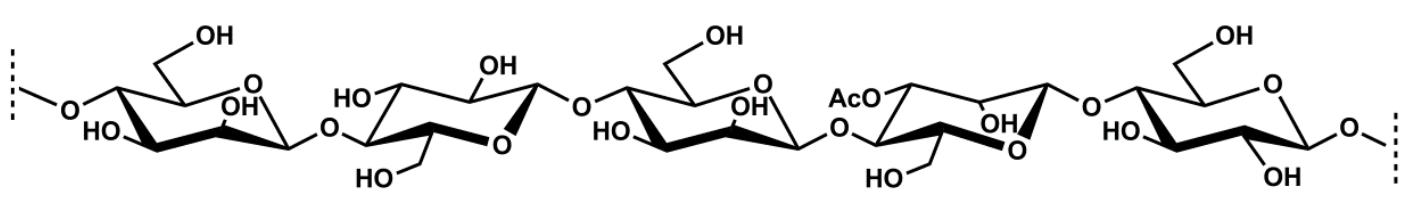

GM

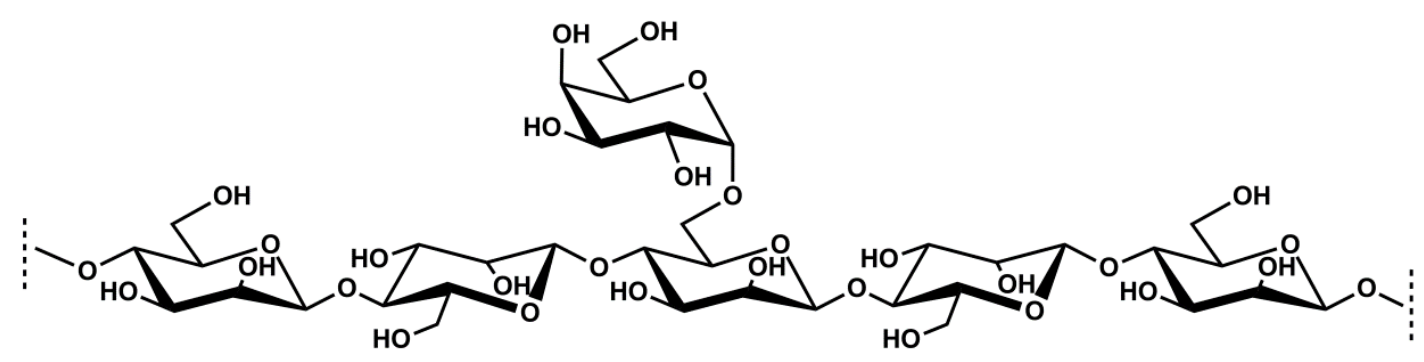

B

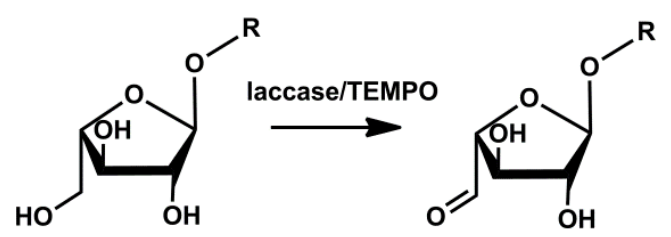

Figure 1. A Structures of the polysaccharides studied. $A X=$ wheat arabinoxylan, $\mathrm{KGM}=$ konjac glucomannan, GM = guar galactomannan. B Example of laccase/TEMPO-mediated oxidation of AX arabinofuranosyl residue, where the primary hydroxyl group is converted to a carbonyl group $(\mathrm{R}=$ the backbone of $\mathrm{AX})$.

\subsection{Molar mass and hydrogel formation}

Oxidized polysaccharide samples were reduced with $\mathrm{NaBH}_{4}$ to prevent crosslinking and to reveal their true molecular masses after the oxidation treatment. Molar masses, determined by sizeexclusion chromatography both for oxidized samples and for the native counterparts, were compared with each other. All the oxidized samples had similar type of elution profiles as shown for AX-ox in Figure 2 (elution profiles for KGM-ox and GM-ox in Supplementary data, Fig. S1). Figure 2 also shows molar masses across the peaks: The peak apex molar masses of native AX and AX-ox were $290000 \mathrm{~g} / \mathrm{mol}$ and $210000 \mathrm{~g} / \mathrm{mol}$, respectively. As can be seen for AX-ox, a peak eluting after the main polysaccharide peak at the retention volumes between $17.5 \mathrm{ml}$ and $19.5 \mathrm{ml}$ 
indicates the presence of small amount of degradation products with the size of the fragments being some tens of $\mathrm{g} / \mathrm{mol}$ (peak apex molar mass of $44000 \mathrm{~g} / \mathrm{mol}$ ). The high-intensity peak which starts to elute at around $20 \mathrm{ml}$ in case of the oxidized sample is expected to originate from the reagents used in the oxidation reaction.

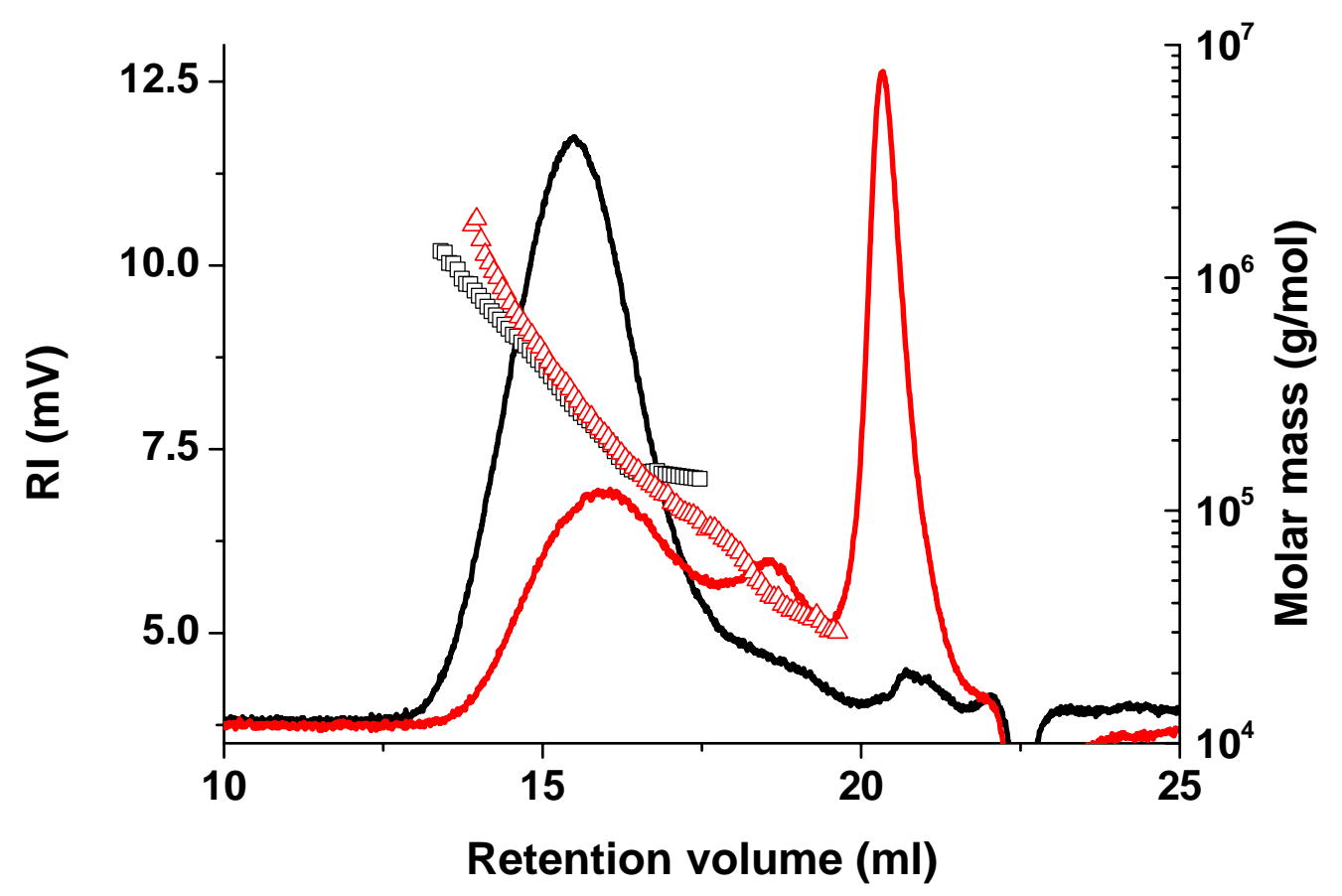

Figure 2. Size exclusion chromatography elution profile and molar mass of AX (black) and AX-ox (red).

Viscoelastic characterization was performed for the solutions of both the unoxidized native polysaccharides and oxidized products. The gelation was studied by oscillation measurement determining the $G^{\prime}$ and $G$ ', moduli as a function of angular frequency. Unoxidized KGM was viscoelastic liquid in the whole frequency range, but AX and GM had a crossover point of G' and G' curves at higher frequencies (Figure 3).

Oxidized polysaccharides appeared as hydrogels at the studied frequency range (Figure 3). The shape of the curves of GM-ox, KGM-ox and AX-ox, with slight increase along with the increasing frequency, and a constant ratio of $\mathrm{G}^{\prime}$ and $\mathrm{G}$ ', independent of the frequency, indicated the samples were true hydrogels. They were flexible but not very highly elastic, such as rubber. 

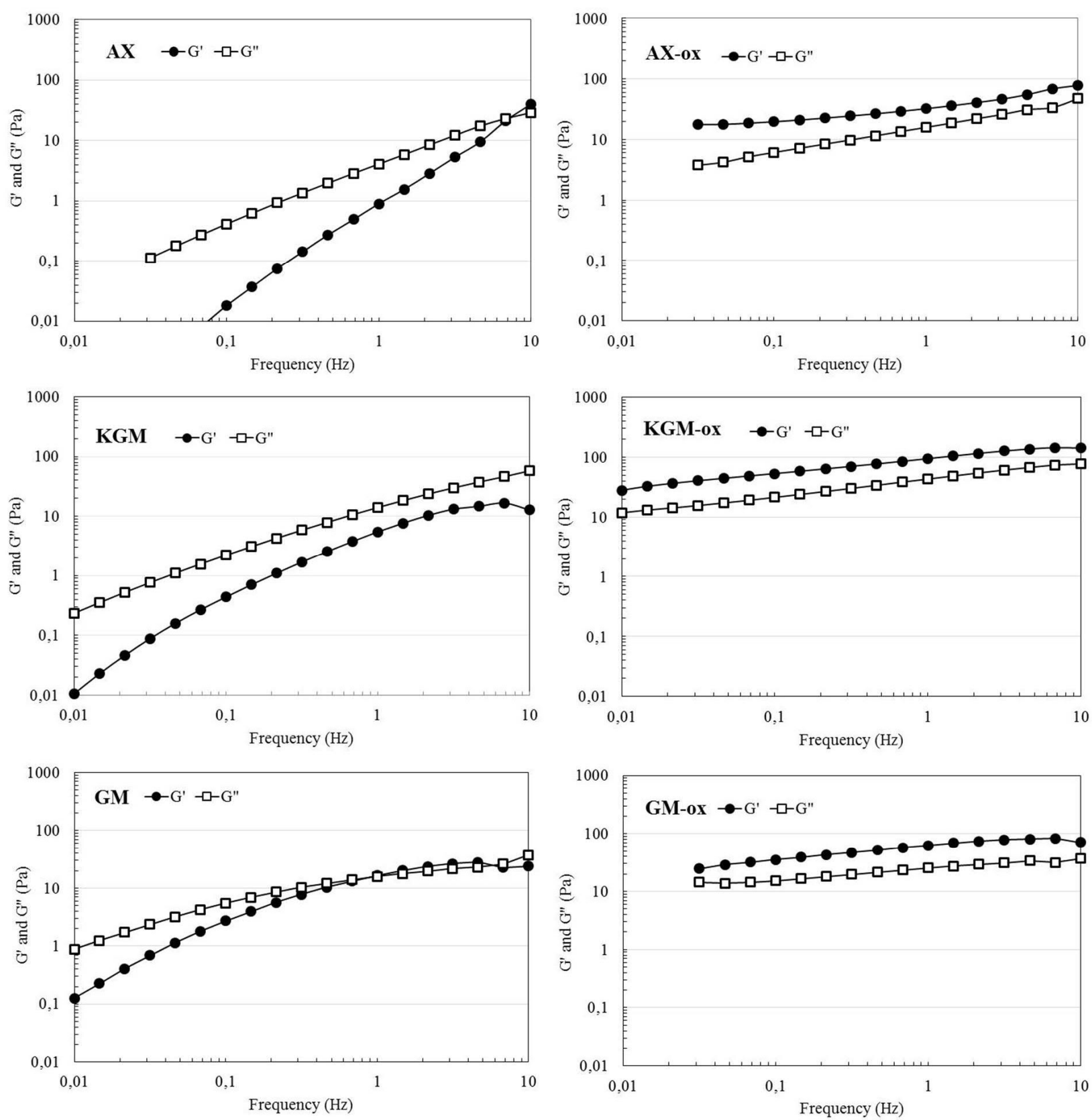

Figure 3. The viscoelastic moduli G' and G', of unoxidized (AX, KGM, GM) and oxidized (AXox, KGM-ox, GM-ox) polysaccharide hydrogel samples further utilized in the aerogel production (DO of total carbohydrates ca. $10 \%$ ).

\subsection{Aerogels}

\subsubsection{Residual TEMPO}

Small amount of TEMPO was still detectable in the aerogel cubes. The amount was ca. $0.2 \mathrm{mg} / \mathrm{g}$ having slight variation in the cube specimens studied. The amount added to the reaction mixture 
was $50 \mathrm{mg} / \mathrm{g}$; thus most of TEMPO had been removed by sublimation during the lyophilization of the aerogel cubes.

\subsubsection{Density}

According to the density determination of aerogels, they were quite lightweight. KGM-ox and GMox aerogels made from $1 \% \mathrm{w} / \mathrm{v}$ hydrogels had densities of ca. $0.020 \mathrm{~g} / \mathrm{cm}^{3}$ (Supplementary data, Table S1). For AX-ox aerogels, made from $2 \% \mathrm{w} / \mathrm{v}$ hydrogels, densities were ca. $0.030 \mathrm{~g} / \mathrm{cm}^{3}$ (Table S1). In all cases, the densities of the reference native polysaccharide samples were somewhat lower. The conventionally frozen samples had slightly higher densities than the ice crystal templated samples.

\subsubsection{Hardness, compressive moduli and resilience}

The cubic aerogel samples of the oxidized polysaccharides and the corresponding unoxidized reference samples were studied with texture analyzer. Hardness, resilience and compressive moduli were calculated from the obtained force-time profile and stress-strain curve.

Ice crystal templated AX-ox was clearly the strongest aerogel of the samples studied (Figure 4 A). The hardness of AX-ox was ca. $70 \mathrm{~N}$, when the sample was compressed by $35 \%$ of its height. Also the conventionally frozen AX-ox was the strongest sample in the compression test when compared to the other conventionally frozen samples. There was a clear difference between the unoxidized and oxidized samples, the unoxidized being weaker than the oxidized aerogels.

The mechanical stiffness of aerogels was studied by determining the compressive moduli of the samples and unoxidized references (Figure 4 B). Again, AX-ox prepared by ice crystal templating had the highest value. The results seemed to follow the same pattern as the in the case of hardness. As expected the unoxidized reference samples had low stiffness. Interestingly, the freezing technique by ice crystal templating seemed to weaken the vertical hardness and compressive modulus of unoxidized samples in the case of AX. The conventionally frozen unoxidized AX was stronger than some of the oxidized samples: conventionally frozen KGM-ox, as well as GM-ox frozen with both of the techniques (Figure 4 A-B).

The aerogel samples did not recover well from the compression. Resiliences were quite low, around $30-40 \%$ (Figure $4 \mathrm{C}$ ). All the unoxidized samples seemed to recover slightly better from the compression than the oxidized samples, probably due to their fluffy and soft structure. Also, in all cases the conventionally frozen samples recovered better than ice crystal templated samples. 

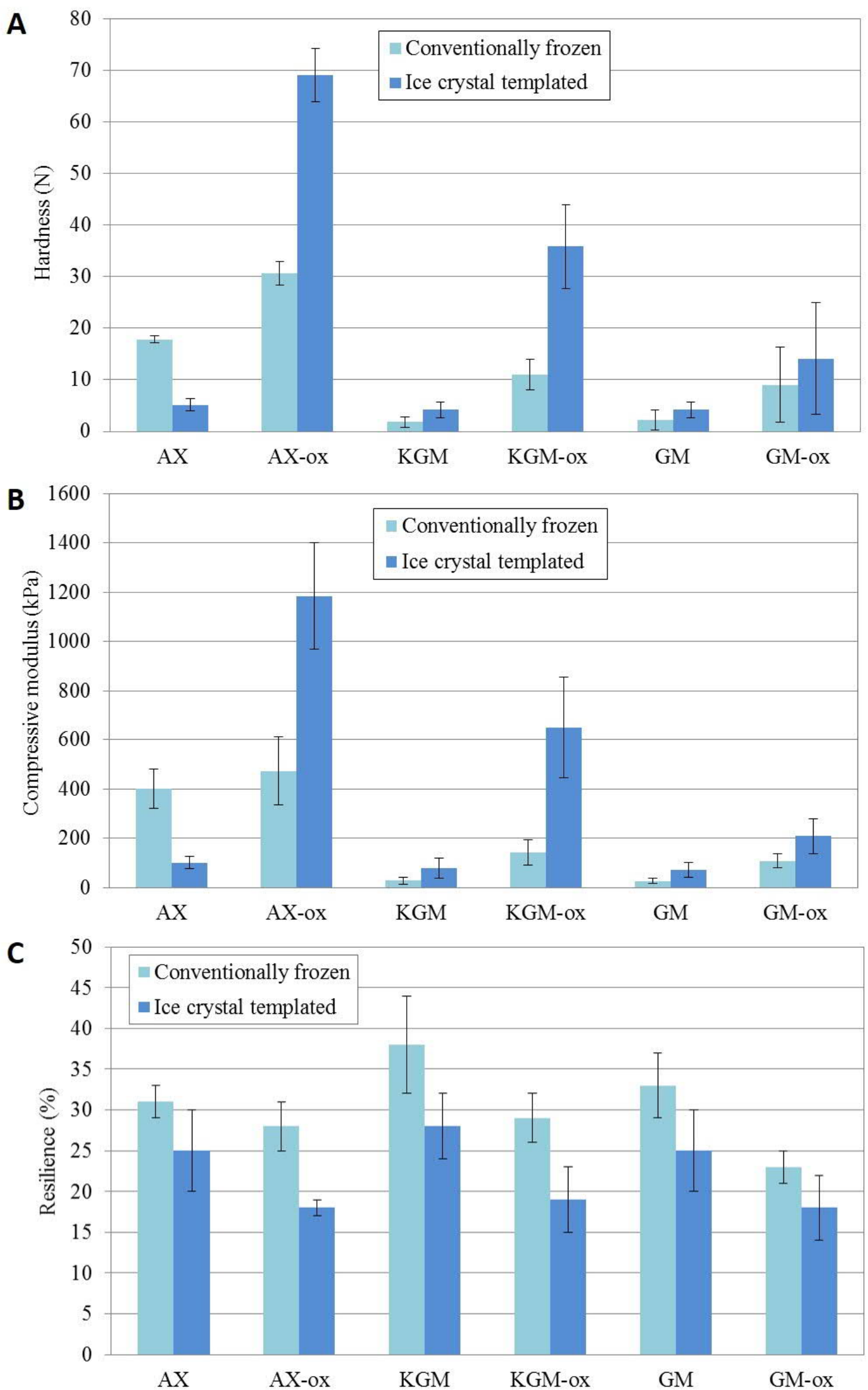
Figure 4. A) The maximum force measured, when the native unoxidized (AX, KGM, GM) and oxidized (AX-ox, KGM-ox, GM-ox) cubic polysaccharide aerogel samples were compressed by $35 \%$ of height in vertical direction. B) The compressive moduli for the similar samples. C) The resilience of the similar samples. The analyses were based on 10 replicates and the error bars indicate standard deviations.

\subsubsection{Water absorption}

The water absorption capacity of cubic aerogel samples was studied at 1, 3 and $24 \mathrm{~h}$ (Table 3). Reference unoxidized samples from native polysaccharide dissolved fast after placing into water. Some differences were observed between conventionally frozen and ice crystal templated samples. Ice crystal templated GM-ox absorbed the highest amount of water after $24 \mathrm{~h}$, almost 40 times the initial weight. Only ice crystal templated AX-ox and GM-ox seemed to fully retain their structure.

Table 3. The water absorption of cubic aerogel samples of both conventionally frozen and ice crystal templated oxidized wheat arabinoxylan (AX-ox), konjac glucomannan (KGM-ox), and guar galactomannan (GM-ox).

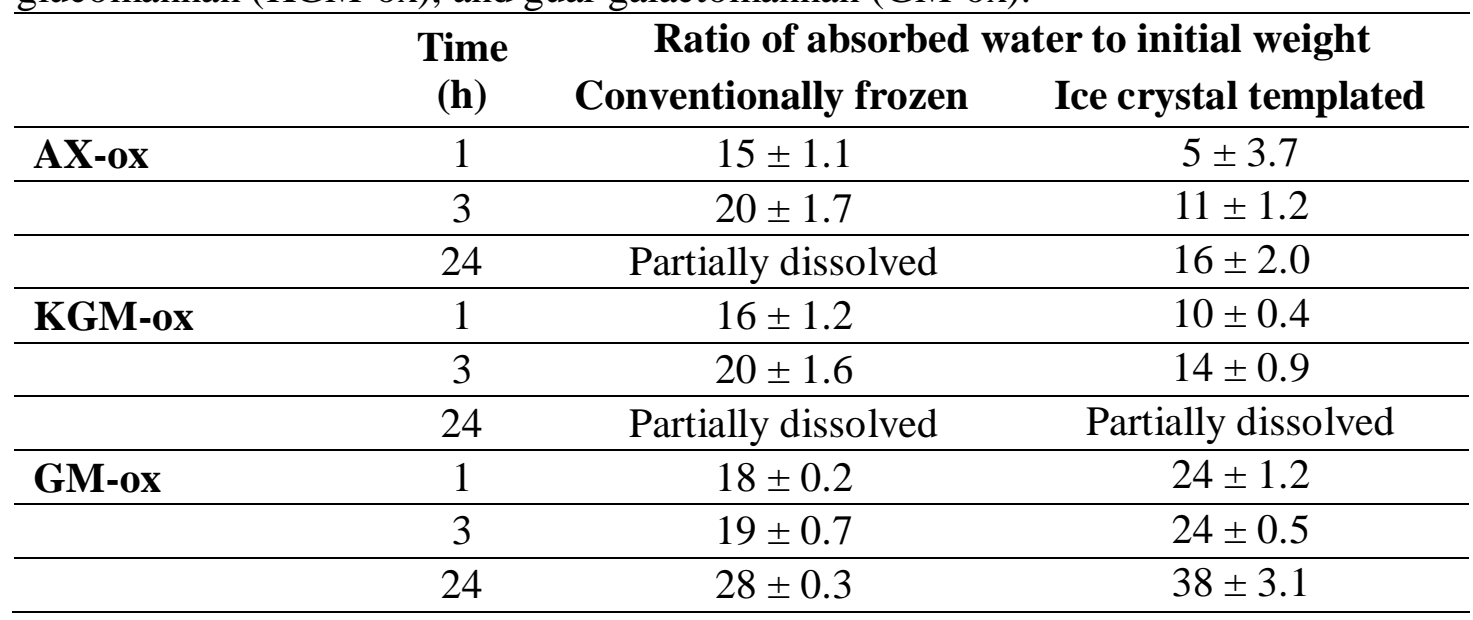

\subsubsection{Morphology}

The cross sections and vertically sliced surfaces of aerogels were imaged by SEM. Ice crystal templated AX-ox formed long and narrow pores, having the width of ca. 50-100 $\mu \mathrm{m}$ and length of a few hundred $\mu \mathrm{m}$ (Figure 5, A a-b), and conventionally frozen AX-ox contained similar structures, but the pore dimensions were much bigger (Figure 5, B a-b). The side views revealed the length of the structures in the vertical direction (Figure 5, A c-d and B c-d). The pore walls of both AX-ox specimens (ice crystal templated and conventionally frozen) were covered with structures looking like craters.

There was a clear difference between ice crystal templated KGM-ox (Figure 5, A e-h) and the conventionally frozen sample (Figure 5, B e-h). The pore diameter of the ice crystal templated 
aerogel was considerably smaller, ca. 50-100 $\mu \mathrm{m}$ (Figure 5, A e-f). Inside the pores, structures resembling spider's web were observed. The thin web was seen in both horizontally (Figure 5, A f) and vertically (Figure 5, A h) sliced samples. According to attempts to focus the microscope closer onto the thin structure, the thickness of a cord of the web was estimated to be 0.01-0.1 $\mu \mathrm{m}$ (Figure 5 C). In the conventionally frozen aerogel, no such web was found (Figure 5, B e-h). The surfaces of the cavities were covered with dots looking like stain, probably due to unsuccessful coating with Pt.

GM-ox resembled the aerogel produced by oxidation with galactose oxidase followed by lyophilisation [Mikkonen et al., 2014], as the structure consisted of similar type of honeycomb (data not shown). The pore size was approximately $25-50 \mu \mathrm{m}$ in the ice crystal templated sample specimens and $100-250 \mu \mathrm{m}$ in the conventionally frozen samples. 

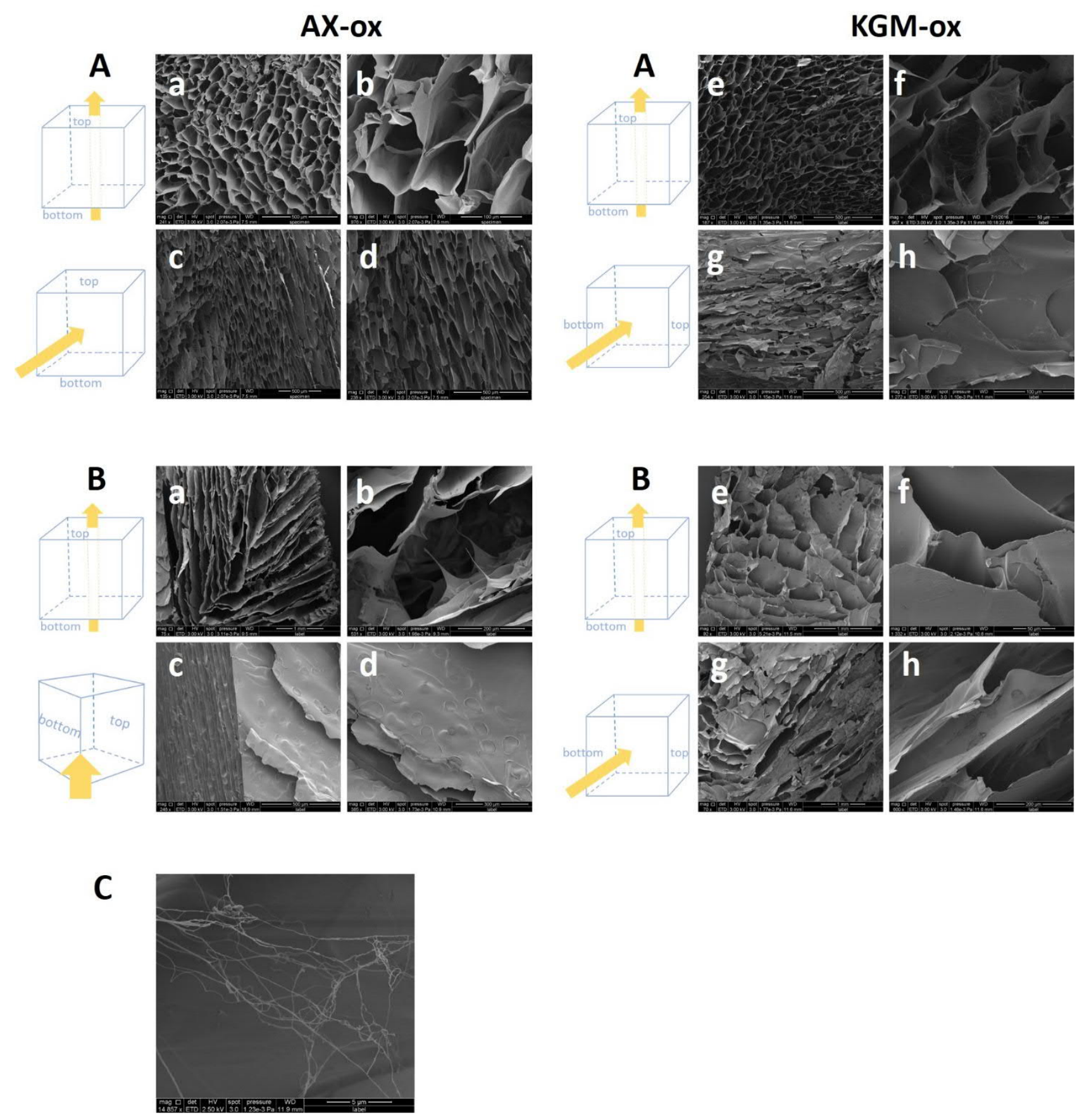

Figure 5. SEM images of $A X-o x(A-B$ a-d) and KGM-ox (A-B e-h) aerogels. A: Ice crystal templated samples. B: Conventionally frozen samples. Samples sliced horizontally; view towards the top (a-b, e-f). Samples sliced vertically; (c-d, g-h). Viewing direction of the cubes shown by the yellow arrow. C: Spider-web structure inside ice crystal templated KGM-ox aerogel pores.

\section{Discussion}

We found the GC-MS method earlier developed for the determination of DO of galactose oxidase -catalyzed reaction also broadly suitable for the analysis of the laccase/TEMPO -mediated 
oxidation products of different polysaccharides. Merlini et al. (2015) have studied laccase/TEMPO oxidized galactomannans (guar, sesbania, locust bean and fenugreek gums) by NMR, but encountered similar problems as we have reported or observed earlier for C-6 aldehyde products: overlapping of chemical shifts, disappearance of aldehyde chemical shifts due to hydrate formation, and poor solubility of some of the products in NMR solvents [Merlini et al., 2015; Parikka and Tenkanen, 2009; Parikka et al., 2010]. By the GC-MS method the oxidized monosaccharide units were reliably identified from the GC chromatogram and corresponding mass spectra.

In the TEMPO-mediated reaction utilizing chemical secondary oxidants such as, $\mathrm{NaBr} / \mathrm{NaClO}$, and resulting in uronic acids, the primary hydroxyls of GM were efficiently oxidized [Parikka et al., 2012], however, the GM samples were partially enzymatically hydrolyzed for the study and thus not fully comparable with this study. For example, all free (not carrying galactosyl side groups) mannosyl units were oxidized to uronic acids [Parikka et al., 2012].

The DOs of the products varied after the individual oxidation reactions. After $24 \mathrm{~h}$ reaction the viscosity started to decrease (data not shown), as also observed in another study [Merlini et al., 2015]. The GC-chromatograms of these samples sometimes showed minor peaks which could be identified as the uronic acid products, thus the further oxidation of the aldehydes (in their hydrate form) [Parikka and Tenkanen, 2009], canceling the ability to crosslink, might have caused the change. Another possibility is the degradation of the polymer chains [Kato et al., 2003]. According to SEC, some degradation had occurred when the reaction was stopped at $24 \mathrm{~h}$, as e.g. in the case of AX-ox, polymers with molar mass of ca. $40 \mathrm{kDa}$ were present in addition to the main product of $210 \mathrm{kDa}$, however, that did not prevent hydrogel formation in the present study. Aggregate formation due to crosslinking also could be an explanation to the decrease of viscosity.

The possible presence of feruloyl substituents in wheat AX might influence the crosslinking, as laccase has been reported to oxidize and dimerize the feruloyl moieties [Selinheimo et al, 2006; Escalante et al., 2013; Iravani et al.; 2011]. These crosslinks were ruled out in this study, as AX used had very low or none feruloyl residues (according to HPLC measurement [Lehtonen et al., 2016] alkali released ferulic acid was under detection limit; data not shown). However, the glucosyl units, present in glucan impurity of AX [Virkki et al., 2008], were oxidized, and might have a role in creating crosslinks as well.

Earlier hydrogels obtained from KGM, prepared by deacetylation, are reported chewy [Takigami, 2009]. The laccase/TEMPO reaction was conducted at slightly acidic $\mathrm{pH}$ and thus no deacetylation was expected, neither seemed the product possess chewiness. The storage modulus of KGM-ox hydrogel reported here was approximately on the same level as that of a borax-crosslinked KGM hydrogel [Gao et al., 2008]. Also the borax-crosslinking method was not based on 
deacetylation, and the experiment was conducted with the same concentration $(1 \% \mathrm{w} / \mathrm{v})$. Thus it seemed that with this type of crosslinking techniques, not involving deacetylation and/or conformation change [Kohyama and Nishinari, 1990] of KGM, chewiness is not achieved.

Similar storage and loss moduli were obtained for the hemiacetal crosslinked AX-ox hydrogel, as have been reported for horse radish peroxidase -treated wheat $\mathrm{AX}(1 \% \mathrm{w} / \mathrm{v})$ [Izydorczyk and Biliaderis, 1992; Izydorczyk 2009]. The relative amount of crosslinks could not be compared to our study as the amount was not estimated for horse radish peroxidase -treated AX. Neither it was determined for the borax-crosslinked KGM, which involved a complicated equilibrium reaction [Gao et al., 2008].

When TEMPO/laccase -oxidized GM hydrogel and aerogel were compared to the products of galactose oxidase -catalyzed oxidation of GM [Parikka et al., 2010, Parikka et al., 2012, Mikkonen et al., 2014], differences were found in the behavior and properties of the products, obviously due to differences in the degree of oxidation and thus differences in the rheological properties. The elastic modulus of GM-ox hydrogel (1\% w/v) was lower in the current study, between 10-100 Pa, when the elastic modulus of the galactose oxidase -oxidized product was $100 \mathrm{~Pa}$ in the whole measurement range - also the DO was clearly higher in the earlier study, 22-28\% [Parikka et al., 2012]. The rheological profile seemed quite similar as reported earlier for GM oxidized with laccase/TEMPO technique [Lavazza et al., 2011]. In the present study the compressive modulus of the aerogel product was slightly lower, and the compressive modulus of unoxidized reference sample seemed to be higher than in the earlier study [Mikkonen et al., 2014].

The densities of the aerogel products were quite similar to those described earlier for polysaccharide aerogels [Mikkonen et al., 2014, Rossi et al., 2016]. The $2 \%$ (w/v) solution of AXox affected the density increasing it roughly up to ca. 1.5 times the densities of samples made from $1 \%(\mathrm{w} / \mathrm{v})$ solutions.

The hardness and mechanical stiffness of the cubic aerogel samples of ice crystal templated AX-ox and KGM-ox were dramatically enhanced when compared to native polysaccharides. In the rheological analysis, the G' and G' moduli of AX-ox and KGM-ox hydrogels were approximately similar to those of GM-ox, in the case of AX-ox slightly lower, thus the rheological study could not be utilized to predict the aerogel properties, as AX-ox had the highest compressive moduli of the samples, ca. $1200 \mathrm{kPa}$. The result on the level of $1200 \mathrm{kPa}$ is clearly approaching the values obtained for nanofibrillated cellulose (NFC) aerogels. For example, $2800 \mathrm{kPa}$ has been reported for NFC aerogel having much higher density than these samples $\left(0.105 \mathrm{~g} / \mathrm{cm}^{3}\right)$ [Sehaqui et al., 2011]. The texture analysis technique utilized here differed from our earlier technique [Mikkonen et al., 2014], and e.g. the speed of compression might influence the obtained values: here, test speed was 
$2.0 \mathrm{~mm} / \mathrm{s}$, and the earlier results for GM-ox were obtained with compression speed of $1.3 \mathrm{~mm} / \mathrm{min}$ [Mikkonen et al., 2014].

It was clear that the ice crystal templated freezing technique was superior to the conventional freezing, if high hardness and mechanical stiffness was pursued. Conventional freezing and lyophilization were utilized in a recent study in the preparation of fenugreek galactomannan aerogel by similar oxidation with laccase/TEMPO -system [Rossi et al., 2016]. Some of the physical properties of the product were determined, and the compressive modulus was very low, only ca. 30 $\mathrm{kPa}$. However, the product was described as spongy, which might explain the difference to the products described here. The formation of ice crystals has been studied at different freezing temperatures, $-15,-25$, and $-40{ }^{\circ} \mathrm{C}$, and the temperature was found to greatly affect the morphology, freezing at $-25{ }^{\circ} \mathrm{C}$ giving the most uniform ice crystal size [Ni et al., 2016].

In the case of unoxidized native $\mathrm{AX}$, the conventionally frozen samples had higher hardness and mechanical stiffness than the ice crystal templated samples. Apparently in this case the crosslinking ability had important role in the structure formation during ice crystal templating; and lacking that, the unoriented polysaccharides formed a stiffer structure. The unoxidized samples recovered better from the compression than the oxidized samples, and in every case, the conventionally frozen samples had higher resilience. This indicated the oriented structures formed in the ice crystal templating suffered more serious damage in the compression thus preventing the recovery, whereas the unoriented polysaccharide chains were able to maintain the structure better. However, the resiliences were quite low for each sample.

KGM-ox and conventionally frozen AX-ox aerogels were found to partially dissolve during the water absorption experiment. Ice crystal templated AX-ox had not dissolved after $24 \mathrm{~h}$ experiment and had absorbed water ca. 16 times initial weight, which is clearly lower than the absorption of a supercritically dried, feruolyl-crosslinked AX aerogel, which was reported to absorb ca. 40 times the initial weight [Escalante et al.; 2013]. Earlier, we have observed values such as, 42 times the initial weight for hemiacetal-crosslinked GM-ox [Mikkonen et al., 2014], thus the ice crystal templated GM-ox prepared here had almost similar value as the galactose oxidase -oxidized GM-ox. Aerogels that start to dissolve in water after certain time might have potential in applications in controlled release of some active components or compounds.

Interestingly, AX-ox consisted of narrow but long pores, prepared by both conventional freezing and ice crystal templating. Ice crystal templating resulted narrower pores and cavities. The structure of ice crystal templated KGM-ox consisted of uniformly distributed somewhat round but flat pores which were considerably smaller than in the conventionally frozen samples. Thus the structures of AX-ox and KGM-ox clearly differed from each other. Most probably, the more thin 
and oriented structure made the ice crystal templated AX-ox stronger than conventionally frozen sample, similarly with the effect of difference in pore size in the case of KGM-ox. When AX-ox aerogel was compared to supercritically dried AX aerogel [Escalante et al., 2013], the pore size of our product was much bigger, as expected [Ghafar et al., 2017]. AX hydrogel (2 \% w/v, containing caffeine), was lyophilized in an earlier study, but the pores of the product were totally different to our results, looking like a honeycomb structure [Iravani et al., 2011]. The source of AX utilized in the study was not wheat but maize bran, which might affect the results. In the case of KGM, similar looking pore structure has been found in lyophilized KGM-glyceryl monostearate aerogels, where relatively round pores with a diameter of ca. $100 \mu \mathrm{m}$ were observed, however, as the aerogels contained also other components than KGM, the results are not directly comparable [Ni et al., 2016]. According to SEM, GM-ox had approximately similar structure as our earlier product obtained from galactose oxidase-catalyzed oxidation [Mikkonen et al., 2014]. Thus the oxidation technique itself seems not to have an effect on the inner structure.

The small crater structures on AX-ox surfaces may have arisen from air bubble bursting during the lyophilization - by naked eye, clearly more air bubbles were observed in AX-ox hydrogel samples than in the others. The formation mechanism of the spider web structures in KGM-ox remained unclear. Similar looking but more widely distributed webs have been found from lyophilized, TEMPO-oxidized cellulose nanofibril dispersions in water/tert-butyl alcohol [Nemoto et al., 2015].

TEMPO was expected to sublime during lyophilization. TEMPO could be considered as nontoxic reagent, as it can be utilized e.g. in vivo antioxidant determinations [Lademann et al., 2014], however, the possibility that TEMPO remains in the aerogels might affect the applications of the products, for example, due to its unpleasant smell. The residual amount was discussed in one of the earlier reports of this aerogel preparation method, where elemental analysis showed the presence of nitrogen in lyophilized fenugreek gum aerogels, but laccase and TEMPO as the nitrogen sources could not be differentiated [Rossi et al., 2016]. Most probably, supercritical drying, instead of lyophlilization, would ensure total disappearance of the reagent from the aerogel. Investigating the effect of supercritical drying on the structure and properties of the products would also constitute an interesting further study.

\section{Conclusions}

We presented the formation of novel AX and KGM hydrogels and aerogels prepared by selective chemo-enzymatic method. The products were formed by hemiacetal crosslinking. The mechanical 
properties of the products were dramatically enhanced when compared to native polysaccharides and e.g. the mechanical stiffness was on significantly higher level, than reported earlier for same type of polysaccharide products. Ice crystal templating was successfully utilized as a freezing method, and the stiffness could be increased by using ice crystal templating instead of conventional freezing. The different structural features obtained by these freezing methods could be visualized by SEM. The products might have potential applications in various fields, e.g. food, food packaging, and pharmacological, regarding the mechanical stiffness and behavior in water solutions.

\section{Acknowledgements}

We thank Dr. Ilya Belevich and Electron Microscopy Unit at the Institute of Biotechnology, University of Helsinki, for conducting the SEM analysis, and Doc. Kirsi S. Mikkonen for fruitful conversations and advice concerning the aerogel properties. Academy of Finland is acknowledged for financial support (project number 281628).

\section{References}

Bragd, P. L.; van Bekkum, H.; Besemer, A. C. Top. Catal., 2004, 27, 49-66.

Buchtová, N.; Budtova, T., Cellulose, 2016, 23, 2585-2595.

Escalante, J. M.; Carvajal-Millan, E.; Miki-Yoshida, M.; Alvarez-Contreras, L.; Toledo-Guillén, A. R.; Lizardi-Mendoza, J.; Rascón-Chu, A. Molecules, 2013, 18, 5531-5542.

Ghafar, A.; Gurikov, P.; Subrahmanyam, R.; Parikka, K.; Tenkanen, M.; Smirnova, I.; Mikkonen, K. S. Compos. Part A: Appl. S., 2017, 94, 93-103.

Iravani, S.; Fitchett, C. S.; Georget, D. M. R. Carbohydr. Polym., 2011, 85, 201-207.

Isogai, A.; Saito, T.; Fukuzumi, H. Nanoscale, 2011, 3, 71-85.

Izydorczyk, M. S. In Handbook of Hydrocolloids, 2nd ed.; Phillips, G. O., Williams, P. A., Eds.; Woodhead Publishing: Cambridge, UK., 2009; p 653-692.

Izydorczyk, M. S.; Biliaderis, C. G. J. Agric. Food Chem., 1992, 40, 561-568.

Jaušovec, D.; Vogrinčič, R.; Kokol, V. Carbohydr. Polym. 2015,116, 74-85. 
Jetten, J. M.; Van Den Dool, R.T.M.; Van Hartingsveldt, W.; Besemer, A. C. 2000. WO Patent $00 / 50463$

Kato, Y.; Matsuo, R.; Isogai, A. Carbohydr. Polym. 2003, 51, 69-75.

Kohyama, K.; Nishinari, K. In Gums and stabilisers for the food industry. G. O. Phillips, D. J. Wedlock, \& P. A. Williams, Eds; IRL Press at Oxford University Press, Oxford, UK, 1990; p. 459.

Lademann, J.; Darvin, M. E.; Fluhr, J. W.; Meinke, M. C. In Non Invasive Diagnostic Techniques in Clinical Dermatology; Berardesca, E.; Maibach, H.; Wilhelm, K.-P., Eds.; Springer, 2014, p. 203-216.

Lavazza, M.; Formantici, C.; Langella, V.; Monti, D.; Pfeiffer, U.; Galante, Y. M. J. Biotech., 2011, $156,108-116$.

Lehtonen, M.; Teräslahti, S.; Xu, C.; Yadav, M. P.; Lampi, A.-M.; Mikkonen, K. S. Food Hydrocolloid., 2016, 58, 255-266.

Li, J.; Ye, T.; Zhoua, B; Li, B. RSC Adv., 2014, 4, 22251-22254.

Merlini, L.; Boccia, A. C.; Mendichi, R.; Galante, Y. M. J. Biotech., 2015, 198, 31-43.

Mikkonen, K. S.; Parikka, K.; Ghafar, A.; Tenkanen, M. Trends Food Sci. Tech., 2013, 34, 124136.

Mikkonen, K. S.; Parikka, K.; Suuronen, J.-P.; Ghafar, A.; Serimaa, R.; Tenkanen, M. RSC Advances, 2014, 4, 11884-11892.

Mulik, S.; Sotiriou-Leventis, C. In Aerogels Handbook; Aegerter, M. A.; Leventis, N.; Koebel, M. M. (Eds.), Springer, 2011, p. 215-232.

Nemoto, J.; Saito, T.; Isogai, A. Appl. Mater. Interfaces, 2015, 7, 19809-19815.

Ni, X.; Ke, F.; Xiao, M.; Wu, K.; Kuang, Y.; Corke, H.; Jiang, F. Int. J. Biol. Macromol., 2016, 92, 1130-1135.

Nieto, M. B. In Functionalizing Carbohydrates for Food Applications, Embuscado, M. E. (Eds.), DESTech Publications, Lancaster, USA, 2014; p 138

Nishinari, K.; Takemasa, M.; Yamatoya, K.; Shirakawa, M. In Handbook of Hydrocolloids, 2nd ed.; Phillips, G. O., Williams, P. A. (Eds.), Woodhead Publishing: Cambridge, UK, 2009; p 535

Parikka, K; Tenkanen, M. Carbohydr. Res., 2009, 344, 14-20.

Parikka, K.; Master, E.; Tenkanen, M. J. Mol. Catal. B: Enzymatic, 2015, 120, 47-59.

Parikka, K.; Ansari, F.; Hietala, S.; Tenkanen, M. Food Hydrocolloid., 2012, 26, 212-220. 
Parikka, K.; Leppänen, A.-S.; Pitkänen, L.; Reunanen, M.; Willför, S.; Tenkanen, M. J. Agric. Food Chem., 2010, 58, 262-271.

Pierre, G.; Punta, C.; Delattre, C.; Melone, L.; Dubessay, P.; Fiorati, A.; Pastori, N.; Galante, Y. M.; Michaud, P. Carbohydr. Polym. 2017, 165, 71-85.

Pitkänen, L.; Virkki, L.; Tenkanen, M.; Tuomainen, P. Biomacromolecules, 2009, 10, 1962-1969.

Pitkänen, L.; Tuomainen, P.; Mikkonen, K. S.; Tenkanen, M. Carbohydr. Polym., 2011, 86, 1230 1235.

Pircher, N.; Fischhuber, D.; Carbajal, L.; Strau, C.; Nedelec, J.-M., Kasper, C.; Rosenau, T.; Liebner, F. Macromol. Mater. Eng. 2015, 300, 911-924.

Quraishi, S.; Martins, M.; Barros, A. A., Gurikov, P.; Raman, S. P.; Smirnova, I.; Duarte, A. R. C.; Reis, R. L. J. Supercrit. Fluids, 2015, 15, 1-8.

Rodgers, C. J.; Blanford, C. F.; Giddens, S. R.; Skamnioti, P.; Armstrong, F. A.; Gurr, S. J. Trends Biotechnol., 2010, 28, 63-72.

Rossi, B.; Campia, P.; Merlini, L.; Brasca, M.; Pastori, N.; Farris, S.; Melone, L.; Punta, C.; Galante, Y. M. Carbohydr. Polym., 2016, 144, 353-361.

Sehaqui, H.; Zhou, Q.; Berglund, A. L. Compos. Sci. Technol., 2011, 71, 1593-1599.

Selinheimo, E.; Kruus, K.; Buchert, J.; Hopia, A.; Autio, K. J. Cereal Sci., 2006, 43, 152-159.

Sescousse, R.; Gavillon, R.; Budtova, T. Carbohydr. Polym., 2011, 83, 1766-1774.

Takigami, S. In Handbook of Hydrocolloids, 2nd ed.; Phillips, G. O., Williams, P. A. (Eds.), Woodhead Publishing: Cambridge, U.K., 2009; p 889.

Tkalec, G.; Knez, Z.; Novak, Z. J. Supercrit. Fluids, 2015, 106, 16-22.

Tojo, G.; Fernandez, M. I. Oxidation of Primary Alcohols to Carboxylic Acids. A Guide to Current Common Practice. 2007; Springer-Verlag, New York, p. 79-103.

Vielinga, W. C. In Handbook of Hydrocolloids, 2nd ed.; Phillips, G. O., Williams, P. A., Eds.; Woodhead Publishing: Cambridge, UK, 2009; p 228

Viikari, L., Buchert, J., Kruus, K., 1999. Method for modification of cellulose. WO Patent 99/23117

Virkki, L.; Maina, H.; Johansson, L.; Tenkanen, M. Carbohydr. Res., 343, 2008, 521-529 


\section{SUPPLEMENTARY DATA}

Table S1. The densities of cubic aerogel samples of native and oxidized wheat arabinoxylan (AX), konjac glucomannan (KGM), and guar galactomannan (GM). GM and KGM samples were prepared from $1 \%(\mathrm{w} / \mathrm{v})$ hydrogels and AX samples from $2 \%(\mathrm{w} / \mathrm{v})$ hydrogels.

\begin{tabular}{lcc}
\hline & $\begin{array}{c}\text { Density/conventionally } \\
\text { frozen }\left(\mathbf{g} / \mathbf{c m}^{\mathbf{3}}\right)\end{array}$ & $\begin{array}{c}\text { Density/ice crystal } \\
\text { templated }\left(\mathbf{g} / \mathbf{c m}^{\mathbf{3}}\right)\end{array}$ \\
\hline $\mathrm{AX}$ & $0.023 \pm 0.0005$ & $0.021 \pm 0.0004$ \\
\hline $\mathrm{AX}-\mathrm{ox}$ & $0.030 \pm 0.0018$ & $0.029 \pm 0.0020$ \\
\hline $\mathrm{KGM}$ & $0.014 \pm 0.0002$ & $0.012 \pm 0.0002$ \\
\hline $\mathrm{KGM-ox}$ & $0.017 \pm 0.0004$ & $0.017 \pm 0.0010$ \\
\hline $\mathrm{GM}$ & $0.017 \pm 0.0005$ & $0.016 \pm 0.0002$ \\
\hline $\mathrm{GM}-\mathrm{ox}$ & $0.023 \pm 0.0006$ & $0.021 \pm 0.0018$ \\
\hline
\end{tabular}

Figure will be prepared by Leena

Figure S1. Size exclusion chromatography elution profile and molar mass of A) KGM (black) and KGM-ox (red); B) GM (black) and GM-ox (red). 